\title{
Some Properties of Weighted Pseudo almost Periodic Functions
}

\author{
Zhe-Ming Zheng, ${ }^{1}$ Hui-Sheng Ding, ${ }^{1}$ and Gaston M. N'Guérékata ${ }^{2}$ \\ ${ }^{1}$ College of Mathematics and Information Science, Jiangxi Normal University, Nanchang, Jiangxi 330022, China \\ ${ }^{2}$ Department of Mathematics, Morgan State University, 1700 E Cold Spring Lane, Baltimore, MD 21251, USA
}

Correspondence should be addressed to Hui-Sheng Ding; dinghs@mail.ustc.edu.cn

Received 31 August 2013; Revised 14 October 2013; Accepted 5 November 2013

Academic Editor: T. Diagana

Copyright ( 2013 Zhe-Ming Zheng et al. This is an open access article distributed under the Creative Commons Attribution License, which permits unrestricted use, distribution, and reproduction in any medium, provided the original work is properly cited.

\begin{abstract}
Several interesting and new properties of weighted pseudo almost periodic functions are established. Firstly, we obtain an equivalent definition for weighted pseudo almost periodic functions, which shows a close relationship between asymptotically almost periodic functions and weighted pseudo almost periodic functions; secondly, we prove that the space of asymptotically almost periodic functions is always a proper subspace of the space of weighted pseudo almost periodic functions; thirdly, we show that under some cases, the space of weighted pseudo almost periodic functions equals the classical space of pseudo almost periodic functions.
\end{abstract}

\section{Introduction}

The notion of weighted pseudo almost periodic function is introduced by Diagana [1], which is an interesting generalization of the classical Bohr almost periodic function as well as a generalization of the classical pseudo almost periodic function introduced by Zhang [2].

Since the work of Diagana, it has been of great interest for many mathematicians to investigate weighted pseudo almost periodic functions and their applications to evolution equations. There is a large amount of literature on this topic. However, due to the influence of weighted term, the behaviors of weighted pseudo almost periodic functions are more tricky and changeable than those of the classical pseudo almost periodic functions.

It is needed to note that, recently, several authors have made interesting and important contributions on weighted pseudo almost periodic functions. For example, Blot et al. $[3,4]$ established many results including completeness, translation invariance, and composition theorem of weighted pseudo almost periodic functions in the framework of measure theory; Diagana [5] investigated the weighted mean for almost periodic functions; Liang et al. [6] showed that the decomposition of weighted pseudo almost periodic functions is not unique, in general, and that translation invariance implies unique decomposition; Ji and Zhang [7] obtained several results concerning translation invariance and ergodicity of an almost periodic function under a weight; Zhang et al. [8] proved that the space of weighted pseudo almost periodic functions is complete under a new norm, which is different from the supremum norm; Ding et al. [9] presented several basic properties about vector-valued weighted pseudo almost automorphic functions, including equivalence, completeness, translation invariance, composition theorem, and convolution theorem of these functions. In addition, we would like to refer the reader to [10-13] for some other recent development on weighted pseudo almost periodic functions and related topics.

Throughout the rest of this paper, we denote by $\mathbb{N}$ the set of positive integers, by $\mathbb{R}$ the set of real numbers, by $X$ a Banach space, by $\mathrm{BC}(\mathbb{R}, X)$ the set of all bounded continuous functions $f: \mathbb{R} \rightarrow X$, and by $\mathbb{U}$ the set of functions (weights) $\rho: \mathbb{R} \rightarrow[0,+\infty)$, which are locally integrable over $\mathbb{R}$. In addition, for $\rho \in \mathbb{U}$ and $r>0$, we denote

$$
\mu(r, \rho):=\int_{-r}^{r} \rho(t) d t
$$




$$
\begin{gathered}
\mathbb{U}_{\infty}:=\left\{\rho \in \mathbb{U}: \lim _{r \rightarrow+\infty} \mu(r, \rho)=+\infty\right\}, \\
\mathbb{U}_{B}:=\left\{\rho \in \mathbb{U}_{\infty}: \rho \text { is bounded with } \inf _{t \in \mathbb{R}} \rho(t)>0\right\} .
\end{gathered}
$$

Obviously, $\mathbb{U}_{B} \subset \mathbb{U}_{\infty} \subset \mathbb{U}$, with strict inclusions.

Next, let us recall some notions and basic results about almost periodic functions and pseudo almost periodic functions (for more details, see [14-16]).

Definition 1. A set $P \subset \mathbb{R}$ is called relatively dense in $\mathbb{R}$ if there exists a number $l>0$ such that, for all $a \in \mathbb{R},[a, a+l] \cap P \neq \varnothing$.

Definition 2. A continuous function $f: \mathbb{R} \rightarrow X$ is called almost periodic if for every $\varepsilon>0$ there exists a relatively dense set $P(\varepsilon)$ such that

$$
\sup _{t \in \mathbb{R}}\|f(t+\tau)-f(t)\|<\varepsilon
$$

for all $\tau \in P(\varepsilon)$. We denote the set of all such functions by $\operatorname{AP}(X)$.

Definition 3. A continuous function $f: \mathbb{R} \rightarrow X$ is called asymptotically almost periodic if it can be expressed as $f=$ $g+h$, where $g \in \operatorname{AP}(X)$ and $h \in C_{0}(X)$, where

$$
C_{0}(X)=\left\{h \in \mathrm{BC}(\mathbb{R}, X): \lim _{|t| \rightarrow \infty} h(t)=0\right\} .
$$

The set of all such functions will be denoted by $\operatorname{AAP}(X)$.

Lemma 4. The following holds true:

(a) if $f \in A A P(X)$, then $f$ is uniformly continuous on $\mathbb{R}$;

(b) a necessary and sufficient condition for a continuous function $f: \mathbb{R} \rightarrow X$ belonging to $A A P(X)$ is that for every $\varepsilon>0$ there exist a constant $T(\varepsilon)>0$ and $a$ relatively dense set $P(\varepsilon)$ such that

$$
\|f(t+\tau)-f(t)\|<\varepsilon
$$

for all $t \in \mathbb{R}$ with $|t| \geq T(\varepsilon)$ and $\tau \in P(\varepsilon)$ with $|t+\tau| \geq$ $T(\varepsilon)$.

Now, let us recall some notions and basic results about weighted pseudo almost periodic functions. Denote

$$
\begin{aligned}
& \operatorname{PAP}_{0}(X, \rho) \\
& :=\left\{f \in \mathrm{BC}(\mathbb{R}, X): \lim _{r \rightarrow+\infty} \frac{1}{\mu(r, \rho)} \int_{-r}^{r}\|f(t)\| \rho(t) d t=0\right\} .
\end{aligned}
$$

Definition 5 (see [1]). Let $\rho \in \mathbb{U}_{\infty}$. A function $f \in \mathrm{BC}(\mathbb{R}, X)$ is called weighted pseudo almost periodic or $\rho$-pseudo almost periodic if it can be expressed as $f=g+h$, where $g \in \operatorname{AP}(X)$ and $h \in \operatorname{PAP}_{0}(X, \rho)$. The set of such functions will be denoted by $\operatorname{PAP}(X, \rho)$.
Remark 6. If $\rho \equiv 1$, then a $\rho$-pseudo almost periodic function becomes a classical pseudo almost periodic function. So $\operatorname{PAP}(X, 1)$ is just the space of all pseudo almost periodic functions. In addition, it is easy to show that $\operatorname{PAP}(X, \rho)=$ $\operatorname{PAP}(X, 1)$ provided that $\rho \in \mathbb{U}_{B}$.

Definition 7 (see [7]). Let $\rho \in \mathbb{U}_{\infty}$. A set $C \subset \mathbb{R}$ is said to be a $\rho$-ergodic zero set if

$$
\lim _{r \rightarrow+\infty} \frac{\int_{[-r, r] \cap C} \rho(t) d t}{\mu(r, \rho)}=0 .
$$

The following lemma is due to [17, Lemma 3.2] (see also [7]).

Lemma 8. Let $f \in B C(\mathbb{R}, X)$ and $\rho \in \mathbb{U}_{\infty}$. Then $f \in$ $P A P_{0}(X, \rho)$ if and only if, for every $\varepsilon>0, M_{\varepsilon}(f)$ is a $\rho$-ergodic zero set, where $M_{\mathcal{\varepsilon}}(f):=\{t \in \mathbb{R}:\|f(t)\| \geq \varepsilon\}$.

\section{The Relationship between $\operatorname{AAP}(X)$ and $\operatorname{PAP}(\boldsymbol{X}, \rho)$}

In this section, we discuss the relationship between $\operatorname{AAP}(X)$ and $\operatorname{PAP}(X, \rho)$. Firstly, we will establish an equivalent definition for weighted pseudo almost periodic functions.

Theorem 9. Let $f \in B C(\mathbb{R}, X)$ and $\rho \in \mathbb{U}_{\infty}$. Then a necessary and sufficient condition for $f \in \operatorname{PAP}_{0}(X, \rho)$ is that there exists a $\rho$-ergodic zero set $C \subset \mathbb{R}$ such that

$$
\lim _{\substack{|t| \rightarrow \infty \\ t \in \mathbb{R} \backslash C}} f(t)=0
$$

Proof. Consider the following.

Sufficiency. It follows from $\lim _{\substack{|t| \rightarrow \infty \\ t \in \mathbb{R} \backslash C}} f(t)=0$ that, for every $\varepsilon>0$,

$$
\{t \in \mathbb{R} \backslash C:\|f(t)\| \geq \varepsilon\}
$$

is a bounded set. Combining this with the fact that $C$ is a $\rho$ ergodic zero set, we conclude that $M_{\varepsilon}(f)=\{t \in \mathbb{R}:\|f(t)\| \geq$ $\varepsilon\}$ is a $\rho$-ergodic zero set. Then, by Lemma $8, f \in \operatorname{PAP}_{0}(X, \rho)$.

Necessity. Let $f \in \operatorname{PAP}_{0}(X, \rho)$. We denote

$$
C_{n}=\left\{t \in \mathbb{R}:\|f(t)\|>\frac{1}{n}\right\}, \quad n \in \mathbb{N} .
$$

Then, by Lemma 8 , every $C_{n}$ is a $\rho$-ergodic zero set. Next, we divide the remaining proof into three steps.

Step 1. There exists an increasing sequence $\left\{T_{n}\right\}_{n=1}^{\infty} \subset \mathbb{R}$ with $\lim _{n \rightarrow \infty} T_{n}=\infty$, satisfying that, for every $n \in \mathbb{N}$ and $r \geq T_{n}$, there hold

$$
\begin{gathered}
\frac{\int_{[-r, r] \cap C_{n+1}} \rho(t) d t}{\mu(r, \rho)} \leq \frac{1}{n}, \\
\frac{\sum_{i=1}^{n} \int_{\left[-T_{i}, T_{i}\right] \cap C_{i}} \rho(t) d t}{\mu(r, \rho)} \leq \frac{1}{n} .
\end{gathered}
$$


In fact, for $n=1$, since $C_{1}$ and $C_{2}$ are both $\rho$-ergodic zero sets, there exists $T_{1}>1$ such that, for all $r \geq T_{1}$, there hold

$$
\begin{gathered}
\frac{\int_{[-r, r] \cap C_{2}} \rho(t) d t}{\mu(r, \rho)} \leq 1, \\
\frac{\int_{\left[-T_{1}, T_{1}\right] \cap C_{1}} \rho(t) d t}{\mu(r, \rho)} \leq \frac{\int_{[-r, r] \cap C_{1}} \rho(t) d t}{\mu(r, \rho)} \leq 1 .
\end{gathered}
$$

For $n=2$, by using the fact that $C_{2}$ and $C_{3}$ are both $\rho$-ergodic zero sets, there exists $T_{2}>\max \left\{T_{1}, 2\right\}$ such that, for all $r \geq T_{2}$, there hold

$$
\begin{gathered}
\frac{\int_{[-r, r] \cap C_{3}} \rho(t) d t}{\mu(r, \rho)} \leq \frac{1}{2}, \\
\frac{\sum_{i=1}^{2} \int_{\left[-T_{i}, T_{i}\right] \cap C_{i}} \rho(t) d t}{\mu(r, \rho)} \\
\leq \frac{\int_{\left[-T_{1}, T_{1}\right] \cap C_{1}} \rho(t) d t+\int_{[-r, r] \cap C_{2}} \rho(t) d t}{\mu(r, \rho)} \leq \frac{1}{2} .
\end{gathered}
$$

For $n=k$, by using the fact that $C_{k}$ and $C_{k+1}$ are both $\rho$ ergodic zero, there exists $T_{k}>\max \left\{T_{k-1}, k\right\}$ such that, for all $r \geq T_{k}$, there hold

$$
\begin{gathered}
\frac{\int_{[-r, r] \cap C_{k+1}} \rho(t) d t}{\mu(r, \rho)} \leq \frac{1}{k}, \\
\frac{\sum_{i=1}^{k} \int_{\left[-T_{i}, T_{i}\right] \cap C_{i}} \rho(t) d t}{\mu(r, \rho)} \\
\leq \frac{\sum_{i=1}^{k-1} \int_{\left[-T_{i}, T_{i}\right] \cap C_{i}} \rho(t) d t+\int_{[-r, r] \cap C_{k}} \rho(t) d t}{\mu(r, \rho)} \leq \frac{1}{k} .
\end{gathered}
$$

Continuing by this way, we can get an increasing sequence $\left\{T_{n}\right\}_{n=1}^{\infty}$, which satisfies $\lim _{n \rightarrow \infty} T_{n}=\infty$, (10).

Step 2. $C:=\bigcup_{n=1}^{\infty} \bar{C}_{n}$ is a $\rho$-ergodic zero set, where

$$
\begin{gathered}
\bar{C}_{1}=\left[-T_{1}, T_{1}\right] \cap C_{1}, \\
\bar{C}_{n}=\left(\left[-T_{n}, T_{n}\right] \backslash\left[-T_{n-1}, T_{n-1}\right]\right) \cap C_{n}, \\
n=2,3, \ldots
\end{gathered}
$$

In fact, for every $r>0$, there exists $T_{n}$ such that $r \in$ $\left[T_{n}, T_{n+1}\right)$. Thus, we have

$$
\begin{aligned}
& {[-r, r] } \subset\left[-T_{1}, T_{1}\right] \cup\left(\left[-T_{2}, T_{2}\right] \backslash\left[-T_{1}, T_{1}\right]\right) \\
& \cup \cdots\left(\left[-T_{n}, T_{n}\right] \backslash\left[-T_{n-1}, T_{n-1}\right]\right) \\
& \cup\left([-r, r] \backslash\left[-T_{n}, T_{n}\right]\right), \\
&([-r, r] \cap C) \subset \bar{C}_{1} \cup \cdots \bar{C}_{n} \cup\left([-r, r] \cap \bar{C}_{n+1}\right) .
\end{aligned}
$$

Combining this with (10), we conclude that

$$
\begin{aligned}
& \frac{\int_{[-r, r] \cap C} \rho(t) d t}{\mu(r, \rho)} \\
& \quad \leq \frac{\sum_{i=1}^{n} \int_{\bar{C}_{i}} \rho(t) d t}{\mu(r, \rho)}+\frac{\int_{[-r, r] \cap \bar{C}_{n+1}} \rho(t) d t}{\mu(r, \rho)} \\
& \quad \leq \frac{\sum_{i=1}^{n} \int_{\left[-T_{i}, T_{i}\right] \cap C_{i}} \rho(t) d t}{\mu(r, \rho)}+\frac{\int_{[-r, r] \cap C_{n+1}} \rho(t) d t}{\mu(r, \rho)} \\
& \leq \frac{2}{n},
\end{aligned}
$$

which means that $C$ is a $\rho$-ergodic zero set.

Step 3. $\lim _{\substack{|t| \rightarrow \infty \\ t \in \mathbb{R} \mid C}} f(t)=0$.

In fact, for every $n \in \mathbb{N}$ and $t \in \mathbb{R} \backslash C$ with $|t|>T_{n}$, there exists $k \geq n$ such that

$$
t \in\left[-T_{k+1}, T_{k+1}\right] \backslash\left[-T_{k}, T_{k}\right],
$$

which yields that $t \notin C_{k+1}$, and thus

$$
\|f(t)\|<\frac{1}{k+1}<\frac{1}{n} .
$$

This completes the proof.

Remark 10. In Theorem 9, we can choose $C$ to be an open set. In fact, letting

$$
\bar{C}=C \bigcup\left(\bigcup_{n=1}^{\infty}\left(T_{n}, T_{n}+\delta_{n}\right)\right) \bigcup\left(\bigcup_{n=1}^{\infty}\left(-T_{n}-\delta_{n},-T_{n}\right)\right),
$$

where every $\delta_{n}$ is a sufficiently small positive constant satisfying

$$
\int_{T_{n}}^{T_{n}+\delta_{n}} \rho(t) d t+\int_{-T_{n}-\delta_{n}}^{-T_{n}} \rho(t) d t<\frac{1}{2^{n}}, \quad n \in \mathbb{N},
$$

it is easy to prove that $\bar{C}$ is an open set and a $\rho$-ergodic zero set. Moreover, obviously, we have $\lim _{|t| \rightarrow \infty} f(t)=0$.

$$
t \in \mathbb{R} \mid \bar{C}
$$

Next, we present an equivalent definition of weighted pseudo almost periodic functions, which establishes a close relationship between asymptotically almost periodic functions and weighted pseudo almost periodic functions.

Theorem 11. Let $f \in B C(\mathbb{R}, X)$ and $\rho \in \mathbb{U}_{\infty}$. Then a necessary and sufficient condition for $f \in P A P(X, \rho)$ is that there exist $\bar{f} \in A A P(X)$ and a $\rho$-ergodic zero set $C \subset \mathbb{R}$ such that

$$
\bar{f}(t)=f(t), \quad t \in \mathbb{R} \backslash C .
$$

Proof. The sufficiency part is easy to prove. We only give the proof for the necessity part. Let $f \in \operatorname{PAP}(X, \rho)$. Then, there 
exist $g \in \mathrm{AP}(X)$ and $h \in \operatorname{PAP}_{0}(X, \rho)$ such that $f=g+h$. It follows from Theorem 9 that there exists a $\rho$-ergodic zero set $C \subset \mathbb{R}$ such that

$$
\lim _{\substack{|t| \rightarrow \infty \\ t \in \mathbb{R} \mid C}} h(t)=0
$$

In addition, by Remark 10, without loss of generality, we can assume that $C$ is an open set. Then, we can conclude that there exists a function $\bar{h} \in C_{0}(X)$ such that $\bar{h}(t)=h(t)$ for all $t \in$ $\mathbb{R} \backslash C$. Letting $\bar{f}=g+\bar{h}$, we have $\bar{f} \in \operatorname{AAP}(X)$, and $\bar{f}(t)=f(t)$ for all $t \in \mathbb{R} \backslash C$. This completes the proof.

Combining Lemma 4 and Theorem 11, we can get the following.

Corollary 12. Let $\rho \in \mathbb{U}_{\infty}$ and $f \in \operatorname{PAP}(X, \rho)$. Then, there exists a $\rho$-ergodic zero set $C \subset \mathbb{R}$ satisfying that, for every $\varepsilon>0$, there are two constants $\delta(\varepsilon), T(\varepsilon)>0$ and a relatively dense set $P(\varepsilon)$ such that

$$
\begin{gathered}
\|f(t+\tau)-f(t)\|<\varepsilon, \\
\tau \in P(\varepsilon), \quad t, t+\tau \notin C \cup[-T(\varepsilon), T(\varepsilon)], \\
\left\|f\left(t_{1}\right)-f\left(t_{2}\right)\right\|<\varepsilon, \quad t_{1}, t_{2} \notin C,\left|t_{1}-t_{2}\right|<\delta(\varepsilon) .
\end{gathered}
$$

Remark 13. For the case of $\rho \equiv 1$, Zhang [18] established a similar result to Corollary 12. But here we use a different approach, and even for the case of $\rho \equiv 1$, Corollary 12 improves the "if part" of [18, Theorem 11]. In fact, in [18], for every $\varepsilon>0$, there exists a 1 -ergodic zero set $C(\varepsilon)$. Here, we find a common ergodic zero set $C$.

In the above, we show that there is a close relationship between asymptotically almost periodic functions and weighted pseudo almost periodic functions. Next, we will show that $\operatorname{AAP}(X)$ is always a proper subspace of $\operatorname{PAP}(X, \rho)$.

Theorem 14. Let $\rho \in \mathbb{U}_{\infty}$. Then

$$
A A P(X) \varsubsetneqq P A P(X, \rho) .
$$

Proof. It is easy to show that $\operatorname{AAP}(X) \subset \operatorname{PAP}(X, \rho)$ since $C_{0}(X) \subset \operatorname{PAP}_{0}(X, \rho)$. So we only need to show that $\operatorname{AAP}(X) \neq \operatorname{PAP}(X, \rho)$. Without loss for generality, we only give the proof for the case of $X=\mathbb{R}$. We divide the remaining proof into three steps.

Step 1. Let $C=\bigcup_{n=1}^{\infty} C_{n}$, where

$$
C_{n}:=\left\{t \in(n-1, n): F(t, n)<\frac{1}{n} F(n-1, n)\right\}, \quad n \in \mathbb{N},
$$

where

$$
F(x, y):=\int_{A(x, y)} \rho(s) d s, \quad A(x, y):=\{s \in \mathbb{R}: x \leq|s| \leq y\}
$$

It is easy to see that every $C_{n}$ is open. Moreover, there are infinitely many nonempty $C_{n}$, and thus we can assume that every $C_{n}$ is nonempty without loss for generality. In addition, we claim that $C$ is a $\rho$-ergodic zero set. In fact, we have the following two cases.

Case I. If $r \in C$, then $r \in C_{[r]+1}$, which yields that

$$
\begin{aligned}
\frac{\int_{[-r, r] \cap C} \rho(t) d t}{\mu(r, \rho)} & \\
\leq & \frac{\int_{[-r, r] \cap C} \rho(t) d t+\int_{A(r,[r]+1)} \rho(t) d t}{\int_{[-r, r]} \rho(t) d t+\int_{A(r,[r]+1)} \rho(t) d t} \\
= & \frac{\int_{[-r, r] \cap C} \rho(t) d t+F(r,[r]+1)}{\mu([r]+1, \rho)} \\
\leq & \frac{\int_{[-r, r] \cap C} \rho(t) d t+(1 /([r]+1)) F([r],[r]+1)}{\mu([r]+1, \rho)} \\
\leq & \frac{\mu([m, \rho)+(2 /(m+1)) \mu([r]+1, \rho)}{\mu([r]+1, \rho)}+\frac{2}{m+1}, \\
\leq & \frac{\sum_{k=1}^{[r]+1} \int_{A(k-1, k) \cap C} \rho(t) d t+(1 /([r]+1)) F([r],[r]+1)}{\mu([r]+1, \rho)} \\
\leq & \frac{\sum_{k=1}^{[r]+1} \int_{C_{k}} \rho(t) d t+(1 /([r]+1)) F([r],[r]+1)}{\mu([r]+1, \rho)} \\
\leq & \frac{\sum_{k=1}^{[r]+1}(1 / k) F(k-1, k)+(1 /([r]+1)) F([r],[r]+1)}{\mu([r]+1, \rho)}
\end{aligned}
$$

where $m<[r]-1$ is a positive integer. Then, we get

$$
\lim _{r \rightarrow+\infty} \frac{\int_{[-r, r] \cap C} \rho(t) d t}{\mu(r, \rho)}=0 .
$$


Case II. If $r \notin C$, then $([r], r] \cap C=\emptyset$, which yields that

$$
\begin{aligned}
& \frac{\int_{[-r, r] \cap C} \rho(t) d t}{\mu(r, \rho)} \\
& \leq \frac{\sum_{k=1}^{[r]} \int_{A(k-1, k) \cap C} \rho(t) d t+\int_{A([r], r) \cap C} \rho(t) d t}{\mu(r, \rho)} \\
& =\frac{\sum_{k=1}^{[r]} \int_{A(k-1, k) \cap C} \rho(t) d t}{\mu(r, \rho)} .
\end{aligned}
$$

Then, similar to Case I, one can also obtain that

$$
\lim _{r \rightarrow+\infty} \frac{\int_{[-r, r] \cap C} \rho(t) d t}{\mu(r, \rho)}=0 .
$$

Thus, $C$ is a $\rho$-ergodic zero set.

Step 2. For every $n \in \mathbb{N}$, noting that $C_{n}$ is open, there exist $t_{n} \in C_{n}$ and $\delta_{n}>0$ such that $\left(t_{n}-\delta_{n}, t_{n}+\delta_{n}\right) \subset C_{n}$. Now, we construct a bounded and continuous function on $\mathbb{R}$ by

$$
\begin{aligned}
& \varphi(t) \\
& =\left\{\begin{array}{cc}
\frac{t-t_{n(n+1) / 2}+\delta_{n(n+1) / 2}}{\delta_{n(n+1) / 2}}, & t \in\left[t_{n(n+1) / 2}-\delta_{n(n+1) / 2},\right. \\
\frac{t_{n(n+1) / 2}+\delta_{n(n+1) / 2}-t}{\delta_{n(n+1) / 2}}, & t \in\left[t_{n(n+1) / 2}, t_{n(n+1) / 2}\right. \\
& \left.+\delta_{n(n+1) / 2}\right], \quad n \in \mathbb{N}, \\
0, & \text { otherwise. }
\end{array}\right.
\end{aligned}
$$

Step 3. $\varphi \in \operatorname{PAP}(X, \rho) \backslash \operatorname{AAP}(X)$.

Since $\{t \in \mathbb{R}: \varphi(t) \neq 0\} \subset C$ and $C$ is a $\rho$-ergodic zero set, we have $\varphi \in \operatorname{PAP}_{0}(X, \rho) \subset \operatorname{PAP}(X, \rho)$. It remains to show that $\varphi \notin \operatorname{AAP}(X)$. We prove it by contradiction, assuming that there exist $\varphi_{1} \in \operatorname{AP}(X)$ and $\varphi_{2} \in C_{0}(X)$ such that $\varphi=\varphi_{1}+\varphi_{2}$. For sufficiently large $n$, since $\varphi_{1} \in \mathrm{AP}(X)$, we can choose

$$
\tau_{n} \in\left[\frac{n(n+1)}{2}-t_{n(n+1) / 2}, \frac{(n+1)(n+2)}{2}-1-t_{n(n+1) / 2}\right]
$$

such that

$$
\left|\varphi_{1}\left(t_{n(n+1) / 2}+\tau_{n}\right)-\varphi_{1}\left(t_{n(n+1) / 2}\right)\right|<\frac{1}{2} .
$$

Moreover, since $\varphi_{2} \in C_{0}(X)$, for sufficiently large $n$, we also have

$$
\left|\varphi_{2}\left(t_{n(n+1) / 2}+\tau_{n}\right)-\varphi_{2}\left(t_{n(n+1) / 2}\right)\right|<\frac{1}{2} .
$$

So, we get

$$
\left|\varphi\left(t_{n(n+1) / 2}+\tau_{n}\right)-\varphi\left(t_{n(n+1) / 2}\right)\right|<1,
$$

which contradicts the fact that

$$
\varphi\left(t_{n(n+1) / 2}\right)=1, \quad \varphi\left(t_{n(n+1) / 2}+\tau_{n}\right)=0 .
$$

This completes the proof.

\section{Equivalence}

Just as noted in Remark 6, we know that $\operatorname{PAP}(X, \rho)=$ $\operatorname{PAP}(X, 1)$ provided that $\rho \in \mathbb{U}_{B}$. Then, there is a natural question:

Does $\operatorname{PAP}(X, \rho)=\operatorname{PAP}(X, 1)$ imply that $\rho \in \mathbb{U}_{B}$ ?

In fact, the above question has a negative answer. For example, recently, it is proved in [7] and [9] (by a different method) that

$$
\operatorname{PAP}\left(X,|\cdot|^{n}\right)=\operatorname{PAP}(X, 1), \quad n \in \mathbb{N} .
$$

In this section, we will make further study on this question. We will prove that for some other $\rho \notin \mathbb{U}_{B}$, there still holds $\operatorname{PAP}(X, \rho)=\operatorname{PAP}(X, 1)$. Firstly, we recall a theorem, which is due to [7, Theorem 4.3].

Theorem 15. Let $\rho_{1}, \rho_{2} \in \mathbb{U}_{\infty}$ and

$\left\{C \subset \mathbb{R}: C\right.$ is a $\rho_{1}$-ergodic zero set $\}$

$$
=\left\{C \subset \mathbb{R}: C \text { is a } \rho_{2} \text {-ergodic zero set }\right\} .
$$

Then $\operatorname{PAP}_{0}\left(X, \rho_{1}\right)=\operatorname{PAP}_{0}\left(X, \rho_{2}\right)$.

Theorem 16. Let $\rho \in \mathbb{U}_{\infty}$ be a periodic function with $\rho(t)>0$ almost everywhere on $\mathbb{R}$. Then $\operatorname{PAP}_{0}(X, \rho)=P A P_{0}(X, 1)$, and thus $P A P(X, \rho)=P A P(X, 1)$.

Proof. It suffices to prove that $\operatorname{PAP}_{0}(X, \rho)=\operatorname{PAP}_{0}(X, 1)$. We divide the remaining proof into two steps.

Step 1. Every $\rho$-ergodic zero set is a 1-ergodic zero set.

Let $C$ be a $\rho$-ergodic zero set. Then, we have

$$
\lim _{r \rightarrow+\infty} \frac{\int_{[-r, r] \cap C} \rho(t) d t}{\mu(r, \rho)}=0 .
$$

Assuming that

$$
\lim _{r \rightarrow+\infty} \frac{\operatorname{mes}([-r, r] \cap C)}{2 r} \neq 0,
$$

then there exist $\varepsilon_{0}>0$ and a sequence of positive numbers $\left\{T_{n}\right\}_{n=1}^{\infty}$, which satisfies $\lim _{n \rightarrow \infty} T_{n}=\infty$, and

$$
\operatorname{mes}\left(\left[-T_{n}, T_{n}\right] \cap C\right)>\frac{2 T_{n}}{\omega} \cdot \varepsilon_{0}
$$

where $\omega$ is a positive periodic of $\rho$. On the other hand, since $\rho(t)>0$ almost everywhere on $\mathbb{R}$, by Lusin's Theorem, there exists a closed set $F \subset[0, \omega]$ with mes $([0, \omega]-F)<\varepsilon_{0} / 2$, such that $\rho$ is continuous on $F$ and $\rho(t)>0$ for all $t \in F$. Let

$$
m_{0}:=\inf _{t \in F} \rho(t)
$$

Then $m_{0}>0$. Then, we have

$$
\operatorname{mes}\left\{t \in[0, \omega]: \rho(t)<m_{0}\right\}<\frac{\varepsilon_{0}}{2} \text {, }
$$


which yields that

$$
\operatorname{mes}\left\{t \in\left[-T_{n}, T_{n}\right]: \rho(t)<m_{0}\right\}<\left(\left[\frac{2 T_{n}}{\omega}\right]+1\right) \cdot \frac{\varepsilon_{0}}{2} \text {. }
$$

Combining this with (41), we get

$$
\begin{aligned}
& \frac{\int_{\left[-T_{n}, T_{n}\right] \cap C} \rho(t) d t}{\mu\left(T_{n}, \rho\right)} \\
& \geq \frac{m_{0} \cdot\left\{\left(2 T_{n} / \omega\right) \cdot \varepsilon_{0}-\left(\left[2 T_{n} / \omega\right]+1\right) \cdot\left(\varepsilon_{0} / 2\right)\right\}}{\int_{\left[-T_{n}, T_{n}\right]} \rho(t) d t} \\
& \geq \frac{m_{0} \cdot\left\{\left(2 T_{n} / \omega\right) \cdot \varepsilon_{0}-\left(\left[2 T_{n} / \omega\right]+1\right) \cdot\left(\varepsilon_{0} / 2\right)\right\}}{\left(\left[2 T_{n} / \omega\right]+1\right) \cdot \int_{0}^{\omega} \rho(t) d t} \\
& \quad \longrightarrow \frac{m_{0} \cdot\left(\varepsilon_{0} / 2\right)}{\int_{0}^{\omega} \rho(t) d t}, \quad n \longrightarrow \infty,
\end{aligned}
$$

which contradicts (39). Therefore, $C$ is a 1-ergodic zero set.

Step 2. Every 1 -ergodic zero set is a $\rho$-ergodic zero set.

Let $C$ be a 1-ergodic zero set. For every $\varepsilon>0$, there exists $\delta>0$ such that, for all set $E \subset[0, \omega]$ with mes $E<\delta$, there holds

$$
\int_{E} \rho(t) d t<\varepsilon
$$

In addition, again by Lusin's Theorem, for the above $\delta>0$, there exists a closed set $F_{\delta} \subset[0, \omega]$ with mes $\left([0, \omega]-F_{\delta}\right)<\delta$, such that $\rho$ is continuous on $F_{\delta}$. Let

$$
M_{\delta}=\sup _{t \in F_{\delta}} \rho(t)
$$

Then $M_{\delta} \in(0,+\infty)$. Recalling that $C$ is a 1-ergodic zero set, for the above $\varepsilon$ and $M_{\delta}$, there exists $r_{\varepsilon}>0$ such that

$$
\lim _{r \rightarrow+\infty} \frac{\operatorname{mes}([-r, r] \cap C)+2 \omega}{2 r}<\frac{\varepsilon}{M_{\delta}}, \quad r \geq r_{\varepsilon} .
$$

Combining (46) and (48), by using the periodicity of $\rho$, we conclude that, for all $r \geq r_{\varepsilon}$, there holds

$$
\begin{aligned}
\int_{[-r, r] \cap C} \rho(t) d t \leq & \sum_{k=0}^{[r / \omega]} \int_{[k \omega, k \omega+\omega] \cap C} \rho(t) d t \\
& +\sum_{k=0}^{[r / \omega]} \int_{[-k \omega-\omega,-k \omega] \cap C} \rho(t) d t \\
\leq & \left(\left[\frac{r}{\omega}\right]+1\right) \cdot 2 \varepsilon \\
& +M_{\delta} \cdot(\operatorname{mes}([-r, r] \cap C)+2 \omega) \\
& \leq\left(\left[\frac{r}{\omega}\right]+1\right) \cdot 2 \varepsilon+2 r \varepsilon,
\end{aligned}
$$

where

$$
\begin{aligned}
\sum_{k=0}^{[r / \omega]} & \int_{[k \omega, k \omega+\omega] \cap C} \rho(t) d t \\
\leq & \sum_{k=0}^{[r / \omega]} \int_{\left[[k \omega, k \omega+\omega]-\left(F_{\delta}+k \omega\right)\right] \cap C} \rho(t) d t \\
& +\sum_{k=0}^{[r / \omega]} \int_{\left[[k \omega, k \omega+\omega] \cap\left(F_{\delta}+k \omega\right)\right] \cap C} \rho(t) d t \\
\leq & \sum_{k=0}^{[r / \omega]} \int_{[k \omega, k \omega+\omega]-\left(F_{\delta}+k \omega\right)} \rho(t) d t \\
& +\sum_{k=0}^{[r / \omega]} M_{\delta} \cdot \operatorname{mes}([k \omega, k \omega+\omega] \cap C) \\
\leq & \sum_{k=0}^{[r / \omega]} \int_{[0, \omega]-F_{\delta}} \rho(t) d t \\
& +M_{\delta} \cdot(\operatorname{mes}([0, r] \cap C)+\omega) \\
\leq & \left(\left[\frac{r}{\omega}\right]+1\right) \cdot \varepsilon+M_{\delta} \cdot(\operatorname{mes}([0, r] \cap C)+\omega)
\end{aligned}
$$

And, similarly,

$$
\begin{aligned}
& \sum_{k=0}^{[r / \omega]} \int_{[-k \omega-\omega,-k \omega] \cap C} \rho(t) d t \\
& \quad \leq\left(\left[\frac{r}{\omega}\right]+1\right) \cdot \varepsilon+M_{\delta} \cdot(\operatorname{mes}([-r, 0] \cap C)+\omega) .
\end{aligned}
$$

Noting that

$$
\int_{-r}^{r} \rho(t) d t \geq 2\left[\frac{r}{\omega}\right] \cdot \int_{0}^{\omega} \rho(t)
$$

we get

$$
\begin{aligned}
\frac{\int_{[-r, r] \cap C} \rho(t) d t}{\int_{-r}^{r} \rho(t) d t} & \leq \frac{([r / \omega]+1) \cdot 2 \varepsilon+2 r \varepsilon}{2[r / \omega] \cdot \int_{0}^{\omega} \rho(t) d t} \\
& \longrightarrow \frac{\omega+1}{\int_{0}^{\omega} \rho(t) d t} \cdot \varepsilon, \quad r \longrightarrow+\infty .
\end{aligned}
$$

Then, by the arbitrariness of $\varepsilon$, we conclude that

$$
\lim _{r \rightarrow+\infty} \frac{\int_{[-r, r] \cap C} \rho(t) d t}{\int_{-r}^{r} \rho(t) d t}=0 ;
$$

that is, $C$ is a $\rho$-ergodic zero set.

Example 17. Let $\rho(t)=\cos ^{2 n}(t)$, where $n \in \mathbb{N}$. Then by Theorem 16, $\operatorname{PAP}(X, \rho)=\operatorname{PAP}(X, 1)$.

Except for the case in Theorem 16, there is some other $\rho$, which satisfies that $\operatorname{PAP}_{0}(X, \rho)=\operatorname{PAP}_{0}(X, 1)$. For example, we have the following. 
Theorem 18. Let

$$
\rho(t)= \begin{cases}\ln |t|, & |t|>1 \\ 0, & |t| \leq 1\end{cases}
$$

Then $\operatorname{PAP}_{0}(X, \rho)=\operatorname{PAP}_{0}(X, 1)$.

Proof. We first show that every 1-ergodic zero set is a $\rho$ ergodic zero set. Let $C$ be a 1 -ergodic zero set. For every $\varepsilon>0$, there exists $R>0$ such that, for all $r>R$,

$$
\operatorname{mes}([-r, r] \cap C)<2 r \cdot \varepsilon \text {. }
$$

Combining this with the fact that $\rho$ is even and increasing on $[0,+\infty)$, we get

$$
\frac{\mu([-r, r] \cap C, \rho)}{\mu([-r, r], \rho)} \leqslant \frac{2 r \ln r \cdot \varepsilon}{2(r \ln r-r+1)}, \quad r>R,
$$

which yields that

$$
\limsup _{r \rightarrow+\infty} \frac{\mu([-r, r] \cap C, \rho)}{\mu([-r, r], \rho)} \leq \varepsilon .
$$

Then, by the arbitrariness of $\varepsilon$, we know that

$$
\lim _{r \rightarrow+\infty} \frac{\mu([-r, r] \cap C, \rho)}{\mu([-r, r], \rho)}=0 ;
$$

that is, $C$ is a $\rho$-ergodic zero set.

Next, let us show that every $\rho$-ergodic zero set is a 1ergodic zero set. Let $C$ be a $\rho$-ergodic zero set. Assuming that

$$
\lim _{r \rightarrow+\infty} \frac{\operatorname{mes}([-r, r] \cap C)}{2 r} \neq 0
$$

then there exist $\varepsilon_{0}>0$ and a sequence of positive numbers $\left\{T_{n}\right\}_{n=1}^{\infty}$, which satisfies $\lim _{n \rightarrow \infty} T_{n}=\infty$, and

$$
\operatorname{mes}\left(\left[-T_{n}, T_{n}\right] \cap C\right)>2 T_{n} \varepsilon_{0} \text {. }
$$

By (61) and the fact that $\rho$ is even and increasing on $[0,+\infty)$, we conclude that

$$
\begin{aligned}
\frac{\mu\left(\left[-T_{n}, T_{n}\right] \cap C, \rho\right)}{\mu\left(\left[-T_{n}, T_{n}\right], \rho\right)} & \geqslant \frac{\mu\left(\left[-T_{n} \varepsilon_{0}, T_{n} \varepsilon_{0}\right], \rho\right)}{\mu\left(\left[-T_{n}, T_{n}\right], \rho\right)} \\
& =\frac{\varepsilon_{0}\left(T_{n} \ln \left(\varepsilon_{0} T_{n}\right)-T_{n}\right)+1}{T_{n} \ln T_{n}-T_{n}+1} \\
& =\frac{\varepsilon_{0}\left(T_{n} \ln T_{n}-T_{n}\right)+T_{n} \varepsilon_{0} \ln \varepsilon_{0}+1}{T_{n} \ln T_{n}-T_{n}+1} .
\end{aligned}
$$

Letting $n \rightarrow \infty$, we get

$$
\liminf _{n \rightarrow \infty} \frac{\mu\left(\left[-T_{n}, T_{n}\right] \cap C, \rho\right)}{\mu\left(\left[-T_{n}, T_{n}\right], \rho\right)} \geq \varepsilon_{0},
$$

which contradicts the fact that $C$ is a $\rho$-ergodic zero set. Thus, $C$ is a 1-ergodic zero set. This completes the proof.

\section{Conflict of Interests}

The authors declare that there is no conflict of interests regarding the publication of this paper.

\section{Acknowledgments}

Ding acknowledges support from the NSF of China (11101192), the Program for Cultivating Young Scientist of Jiangxi Province (20133BCB23009), and the NSF of Jiangxi Province; Zheng acknowledges support from the Graduate Innovation Fund of Jiangxi Province.

\section{References}

[1] T. Diagana, "Weighted pseudo almost periodic functions and applications," Comptes Rendus Mathématique, vol. 343, no. 10, pp. 643-646, 2006.

[2] C. Y. Zhang, "Pseudo-almost-periodic solutions of some differential equations," Journal of Mathematical Analysis and Applications, vol. 181, no. 1, pp. 62-76, 1994.

[3] J. Blot, P. Cieutat, and K. Ezzinbi, "New approach for weighted pseudo-almost periodic functions under the light of measure theory, basic results and applications," Applicable Analysis, vol. 92, no. 3, pp. 493-526, 2013.

[4] J. Blot, P. Cieutat, and K. Ezzinbi, "Measure theory and pseudo almost automorphic functions: new developments and applications," Nonlinear Analysis: Theory, Methods \& Applications, vol. 75, no. 4, pp. 2426-2447, 2012.

[5] T. Diagana, "The existence of a weighted mean for almost periodic functions," Nonlinear Analysis: Theory, Methods \& Applications, vol. 74, no. 12, pp. 4269-4273, 2011.

[6] J. Liang, T.-J. Xiao, and J. Zhang, "Decomposition of weighted pseudo-almost periodic functions," Nonlinear Analysis: Theory, Methods \& Applications, vol. 73, no. 10, pp. 3456-3461, 2010.

[7] D. Ji and C. Zhang, "Translation invariance of weighted pseudo almost periodic functions and related problems," Journal of Mathematical Analysis and Applications, vol. 391, no. 2, pp. 350362, 2012.

[8] J. Zhang, T.-J. Xiao, and J. Liang, "Weighted pseudo almostperiodic functions and applications to semilinear evolution equations," Abstract and Applied Analysis, vol. 2012, Article ID 179525, 15 pages, 2012.

[9] H.-S. Ding, J. Liang, and T.-J. Xiao, "Weighted pseudo almost automorphic functions and WPAA solutions to semilinear evolution equations," Journal of Mathematical Analysis and Applications, vol. 409, no. 1, pp. 409-427, 2014.

[10] S. Abbas, "Pseudo almost automorphic solutions of some nonlinear integro-differential equations," Computers \& Mathematics with Applications, vol. 62, no. 5, pp. 2259-2272, 2011.

[11] J.-H. Liu and X.-Q. Song, "Almost automorphic and weighted pseudo almost automorphic solutions of semilinear evolution equations," Journal of Functional Analysis, vol. 258, no. 1, pp. 196-207, 2010.

[12] H.-S. Ding, G. M. N'Guérékata, and J. J. Nieto, "Weighted pseudo almost periodic solutions for a class of discrete hematopoiesis model," Revista Matemática Complutense, vol. 26, no. 2, pp. 427-443, 2013.

[13] H.-S. Ding, J. Liang, and T.-J. Xiao, "Weighted pseudo almost periodic functions and applications to evolution equations with 
delay," Applied Mathematics and Computation, vol. 219, no. 17, pp. 8949-8958, 2013.

[14] C. Corduneanu, Almost Periodic Functions, Chelsea, New York, NY, USA, 2nd edition, 1989.

[15] T. Diagana, Pseudo Almost Periodic Functions in Banach Spaces, Nova Science, New York, NY, USA, 2007.

[16] C. Zhang, Almost Periodic Type Functions and Ergodicity, Science Press, Beijing, China, 2003.

[17] H.-S. Ding, W. Long, and G. M. N'Guérékata, "A composition theorem for weighted pseudo-almost automorphic functions and applications," Nonlinear Analysis: Theory, Methods \& Applications, vol. 73, no. 8, pp. 2644-2650, 2010.

[18] C. Zhang, "Vector-valued pseudo almost periodic functions," Czechoslovak Mathematical Journal, vol. 47, no. 122, pp. 385394, 1997. 


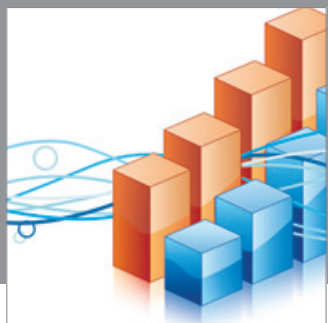

Advances in

Operations Research

mansans

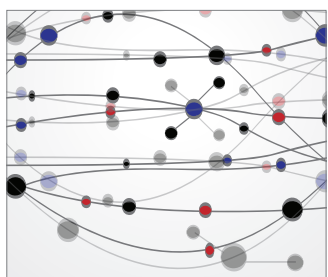

The Scientific World Journal
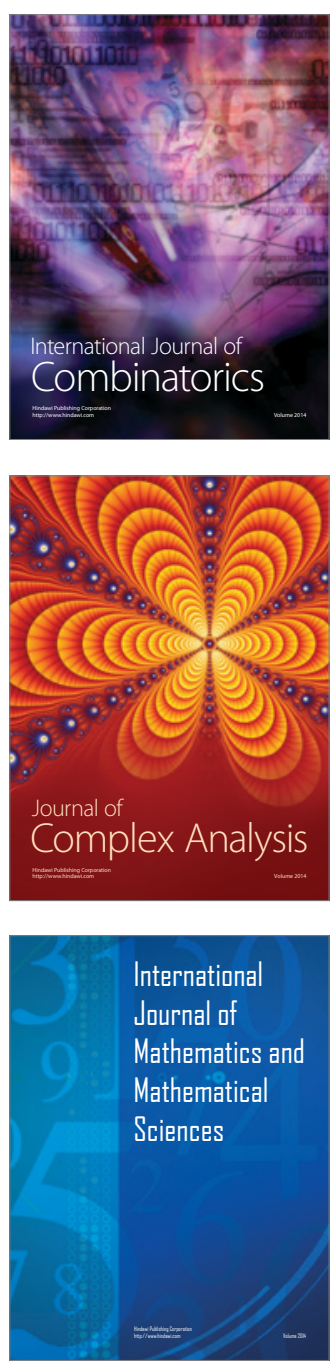
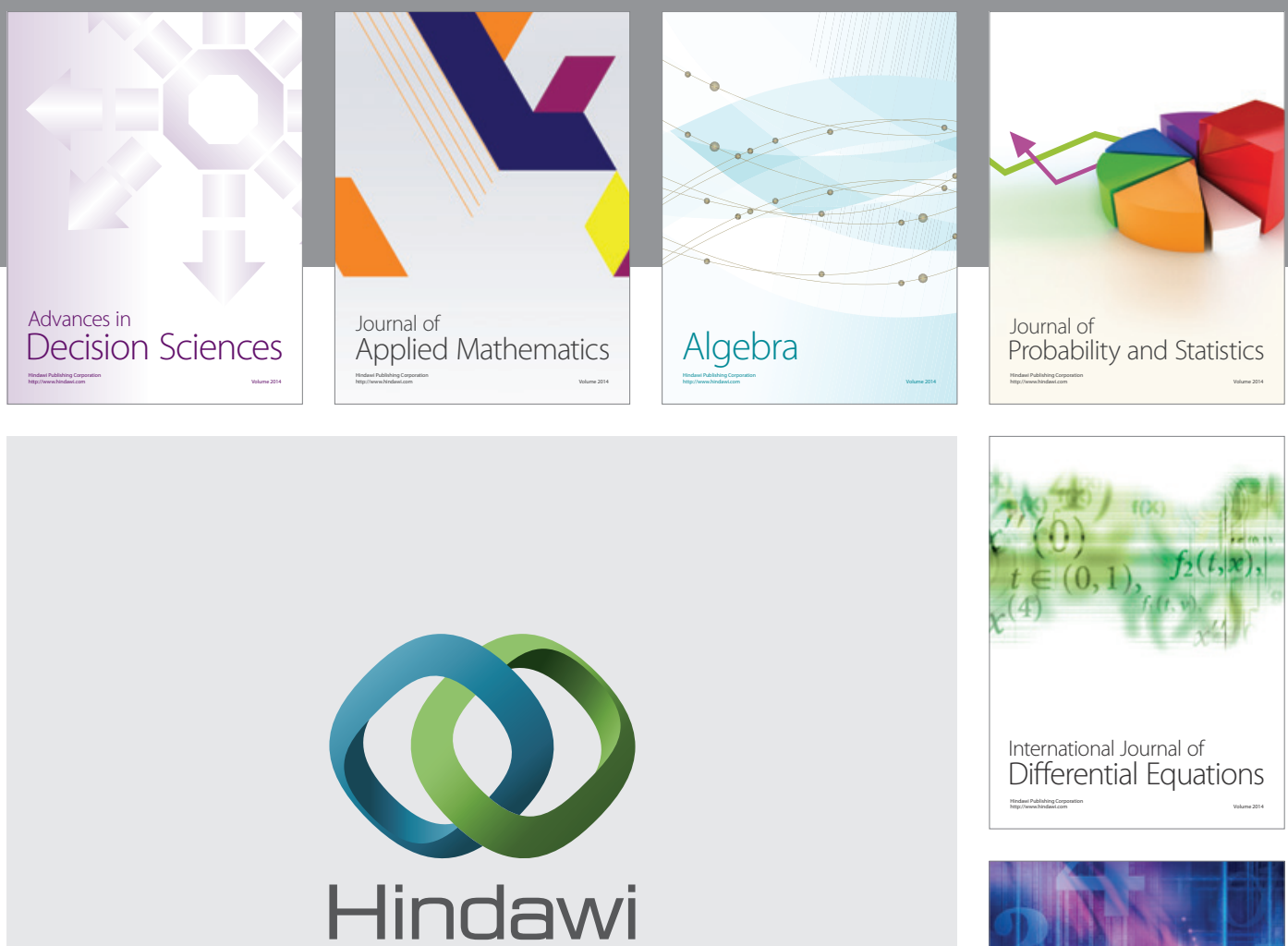

Submit your manuscripts at http://www.hindawi.com
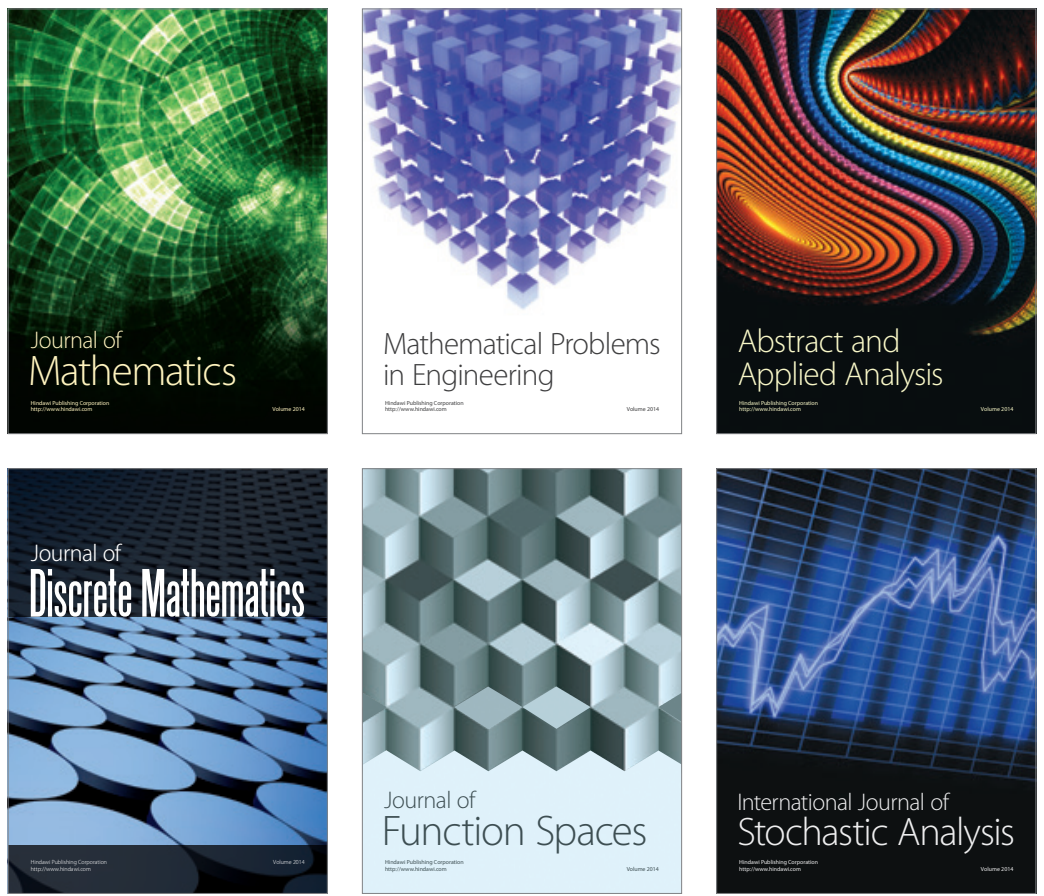

Journal of

Function Spaces

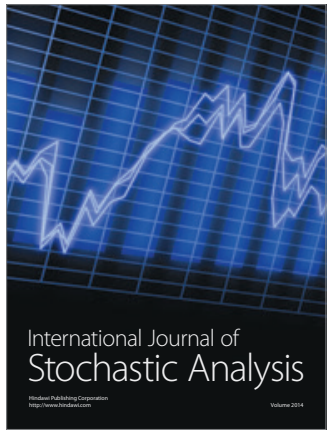

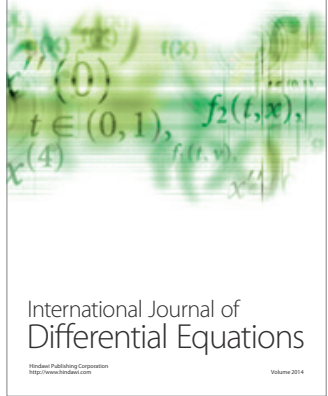
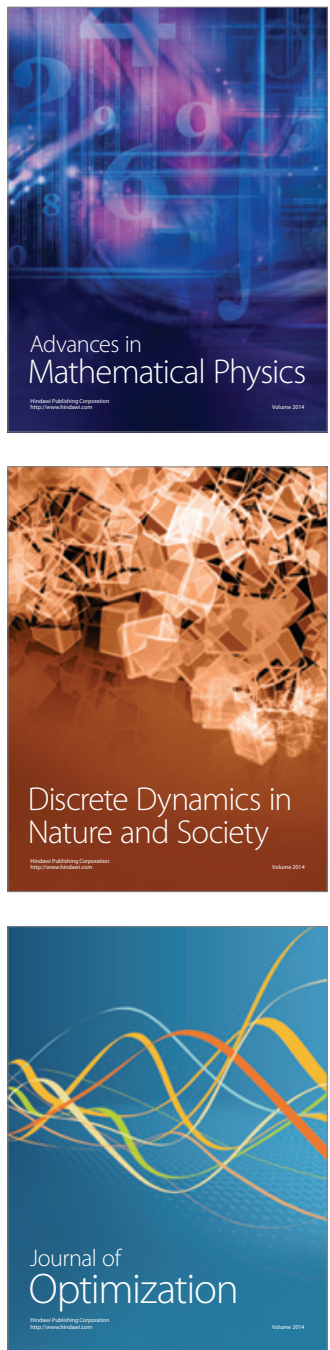